\title{
The 3D Computer Image of the Anterior Corneal Surface
}

\author{
Bo Wang ${ }^{1}$, Xueping Huang ${ }^{2}$, Jinglu Ying ${ }^{3}$, Mingguang Shi ${ }^{3}$ \\ ${ }^{1}$ School of Applied Mathematics, Nanjing University of Finance and Economics, Nanjing, China \\ ${ }^{2}$ Wenzhou Medical College, Wenzhou, China \\ ${ }^{3}$ Department of Ophthalmology, The Second Affiliated Hospital of Wenzhou Medical College, Wenzhou, China \\ Email: bowang@live.com, pshimg@hotmail.com
}

Received 2013

\begin{abstract}
In this paper, we derive a nonlinear equation of corneal asphericity $(Q)$ using the tangential radius of curvature $\left(r_{t}\right)$ on every semi-meridian. We transform the nonlinear equation into the linear equation and then obtain the $Q$-value of corneal semi-meridian by the linear regression method. We find the 360 semi-meridional variation rule of the $Q$-value using polynomial function. Furthermore, we construct a new 3D corneal model and present a more realistic model of shape of the anterior corneal surface.
\end{abstract}

Keywords: Cornea; Computer; Image

\section{Introduction}

It is well established that the anterior surface of the cornea is the major refractive element of the human eye, being responsible for approximately $75 \%$ of the eye's total unaccommodated refractive power [1]. Guillon [2] and Bennett [3] assumed the human cornea to have a conic section which can be described by the Baker's equation: $y^{2}=2 r_{0} x-p x^{2}$ [4]. Here, $p$ describes the asphericity of the corneal section. Cheung [5] calculated the corneal asphericity $(p)$ using the sagittal radius of curvature $\left(r_{s}\right)$ from corneal axial power map according to Bennett's equation: $r_{s}^{2}=r_{0}^{2}+(1-p) y^{2}$ [6]. Schwiegerling [7] used corneal height data from corneal height map and Zernike polynomials to describe the shape of the cornea. Corneal topography is commonly presented as axial power map, tangential power map and height map. To our knowledge, there is no report of investigating the corneal asphericity $(Q, Q=p-1)$ calculation by the tangential radius of curvature $\left(r_{t}\right)$ from tangential power map.

In most previous studies, it has been reported the $Q$ values which is representative of all corneal meridians or the $Q$-values of two principal meridians. Dubbelman [8] measured $k$-values (where $k=Q+1$ ) of six semimeridians $\left(0^{\circ}, 30^{\circ}, 60^{\circ}, 90^{\circ}, 120^{\circ}, 150^{\circ}\right)$ using Scheimpflug photography and modeled the meridional variation of the $k$-value using the $\cos ^{2}$ function. However, it indicated that the $\cos ^{2}$ function is not an adequate model to describe the variation.

Sagittal radius of curvature $\left(r_{s}\right)$ is spherically biased and is not a true radius of curvature [9-11] and it will lead to erroneous result for an asymmetric corneal surface. Tangential radius of curvature $\left(r_{t}\right)$ is a true radius of curvature which can better represent corneal shape and local curvature changes especially in the periphery [12]. In this paper we derive a nonlinear equation of corneal asphericity $(Q)$ using the tangential radius of curvature $\left(r_{t}\right)$ on every semi-meridian for the first time. We obtain the $Q$-value of corneal semi-meridian by the linear regression method and find the 360 semi-meridional variation rule of the $Q$-value using polynomial function. Furthermore, we construct a new 3D model of shape of the anterior corneal surface.

\section{Derivation of the Corneal Model}

The Bausch \& Lomb Orbscan II corneal topographer is used to acquire images of the topography of the right eye of 66 normal young subjects. All subjects have no history of ocular disease and ocular surgery with emmetropic eyes. A series of data point on a semi-meridian are arranged at $0.1 \mathrm{~mm}$ intervals. The interval between two semi-meridians is $1^{\circ}$. The tangential radius of curvature $\left(r_{t}\right)$ and perpendicular distance from the point to optical axis $(y)$ of all data point on a semi-meridian and vertex radius of curvature ( $r_{0}$-value) can be obtained from the raw data of tangential power map of anterior corneal surface.

A three dimensional Cartesian coordinate system is set with its origin at vertex normal to the corneal intersection of the optic axis of the corneal topographer [13]). The $Z$-axis, $Y$-axis, $X$-axis of the coordinate represent the optical axis direction, the vertical direction and the hori- 
zontal direction, respectively. $\theta$ is the angle between the corneal meridian section and the $\mathrm{XOZ}$ plane. The corneal meridian section is located on the $\mathrm{YOZ}$ plane when $\theta=90^{\circ}$. At this time, we assume that the equation of corneal meridian can be correspondingly described by the conic equation: $y^{2}=a_{1} z+a_{2} z^{2}, a_{1}, a_{2} \in R$. This conic equation is an improvement of the Baker's equation [4]. While located on the $\mathrm{XOZ}$ plane when $\theta=0^{\circ}$ and described by the conic equation:

$x^{2}=a_{1} z+a_{2} z^{2}$. For any other angle $\theta$ except for $0^{\circ}$, $90^{\circ}, 180^{\circ}, 270^{\circ}$, the $Y O Z$ plane can be coincided with the corneal meridian section by rotating the coordinate system. Thus the corneal meridian section of any other angle $\theta$ can also be described by the conic equation $y^{2}=a_{1} z+a_{2} z^{2}$ in the new coordinate system (see Section 5.2).

Here let us take corneal meridian section with $\theta=$ $90^{\circ}$ for example, the formula of the curvature of a point on the section can be expressed as $[14,15]$ :

$$
K=\frac{1}{r_{t}}=\frac{\left|y^{\prime \prime}\right|}{\sqrt{\left[1+\left(y^{\prime}\right)^{2}\right]^{3}}}
$$

where $K$ is curvature, $y^{\prime}$ and $y^{\prime \prime}$ are the first and second derivatives with respect to $Z$ which is $Z$-axis coordinate value of the point. Differentiating both sides of the conic equation $y^{2}=a_{1} z+a_{2} z^{2}$ with respect to $Z$, we get

$$
y^{\prime}=\frac{a_{1}+2 a_{2} z}{2 y}, \quad y^{\prime \prime}=\frac{-a_{1}^{2}}{4 y^{3}}
$$

Then by substituting $y^{\prime}$ and $y^{\prime \prime}$ into Equation (1), we obtain:

$$
r_{t}=\frac{4}{a_{1}^{2}}\left(\frac{a_{1}^{2}}{4}+\left(1+a_{2}\right) y^{2}\right)^{\frac{3}{2}}
$$

The conic equation $y^{2}=a_{1} z+a_{2} z^{2}$ can be rewritten as:

$$
\frac{\left(z+\frac{a_{1}}{2 a_{2}}\right)^{2}}{\frac{a_{1}^{2}}{4 a_{2}^{2}}}+\frac{y^{2}}{-\frac{a_{1}^{2}}{4 a_{2}}}=1
$$

Since $Q=-e^{2}$, then by Equation (3) we have $a_{2}=-(1+Q)$.

Finally by substituting $a_{2}=-(1+Q)$ into Equation (2), we obtain

$$
r_{t}=\frac{1}{r_{0}^{2}}\left[r_{0}^{2}-Q y^{2}\right]^{\frac{3}{2}}
$$

\section{Solution to $Q$-Value Calculation Problem}

Since $r_{t}$ is a nonlinear function of $y$ in Equation (4), it is difficult to calculate $Q$-value. To transform the nonlinear problem to the linear one, the Equation (4) is converted to another form which can be written as:

$$
y^{2}=b+c r_{t}^{\frac{2}{3}}
$$

where $b$ and $c$ are constants, a straight line graph of $y^{2}$ (on the ordinate) vs $r_{t}^{\frac{2}{3}}$ (on the abscissa) is plotted. By the linear regression method, we get $b=\frac{r_{0}{ }^{2}}{Q}$ and $c=-\frac{r_{0}^{\frac{4}{3}}}{Q}$, that is, $Q=-\frac{b^{2}}{c^{3}}$. The straight line gives a coefficient of determination $\left(R^{2}\right)$. The $Q$-value of the given semi-meridian is calculated including from the first point at $0.1 \mathrm{~mm}$ to $3.5 \mathrm{~mm}$. Figure 1 illustrates a function scatterplot of perpendicular distance squared versus tangential radius of curvature to the two-thirds power on the nasal horizontal principal semi-meridian of the right eye for subject number 1 .

\section{360 Semi-Meridional Rule of the $Q$-Value}

The corneal zone analyzed is up to diameter $7.0 \mathrm{~mm}$ which is large enough to cover the pupillary area. The near vertical meridians will have a diameter limit imposed by the eyelids and eyelashes. In our earlier study, we found that the semi-meridians which the peripheral points were up to $3.5 \mathrm{~mm}$ were mainly distributed within $50^{\circ}$ of the horizontal including $0^{\circ}-50^{\circ}, 130^{\circ}-180^{\circ}, 181^{\circ}$ $230^{\circ}, 310^{\circ}-359^{\circ}$. The $Q$-value of each semi-meridian in these near horizontal regions was calculated. According to the $Q$-values of the near horizontal semi-meridians, we use regression analysis to model the 360 semi-meridional variation of the $Q$-value and fit the $Q$-value of

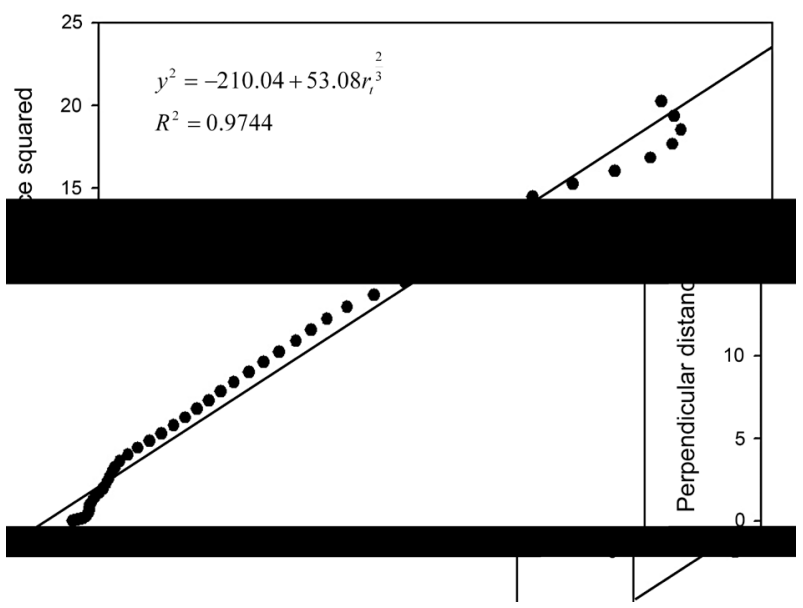

Figure. 1. Scatterplot of perpendicular distance squared versus tangential radius of curvature to the two-thirds power. 
each semi-meridian in the near vertical regions including $51^{\circ}-129^{\circ}, 231^{\circ}-309^{\circ}$. The form of a polynomial function is:

$$
f(x)=p_{0}+p_{1} x+p_{2} x^{2}+p_{3} x^{3}+p_{4} x^{4}+\ldots
$$

where $x$ is semi-meridian angle $\theta$ (degree) and $f(x)$ is corresponding $Q$-value. Here, the degree must be converted to the radian when calculating the polynomial fitting.

To determine which degree polynomial will provide an optimal fit to the 360 semi-meridional variation of the $Q$-value, we calculate the RMS fit error of the polynomial function from $5^{\text {th }}$ degree to $9^{\text {th }}$ degree. We find that the RMS fit error become relatively stable at approximately 0.02 for fits higher than $6^{\text {th }}$ degree.

The 360 semi-meridional variation of the $Q$-value is well fitted using the $7^{\text {th }}$ degree polynomial function for all subjects. Figure 2 shows an example of the variation of the $Q$-value as a function of semi-meridian for subject number 22 with the following $7^{\text {th }}$ degree polynomial function:

Red: Fitted curve of 360 semi-meridional variation of the Q-value.

Figure 3 shows that the majority of right eyes display the goodness of fit $\left(r^{2}\right)$ of polynomial function for all subjects for the asphericity above 0.9 and the median value is 0.94 . The mean RMS fit error of polynomial fit is $0.02 \pm 0.008$.

Figure 4 shows the variation in asphericity with semimeridian region of anterior corneal surface for all subjects. It can be seen that the $Q$-value distribution of anterior corneal surface presents bimodal variation. These two peak values represent the least negative $Q$-values.

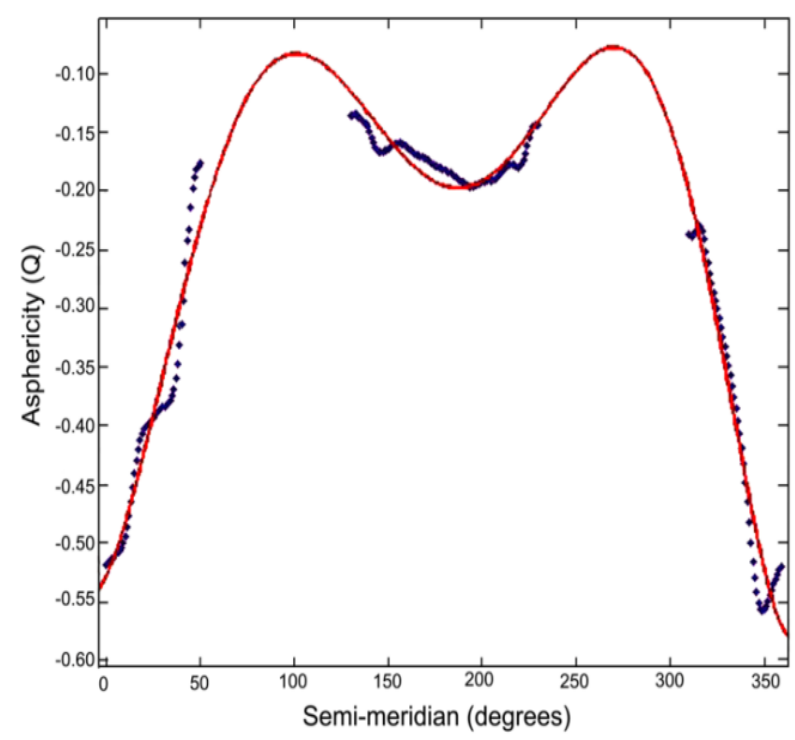

Figure 2. Typical example of the variation of the $Q$-value as a function of semi-meridian.

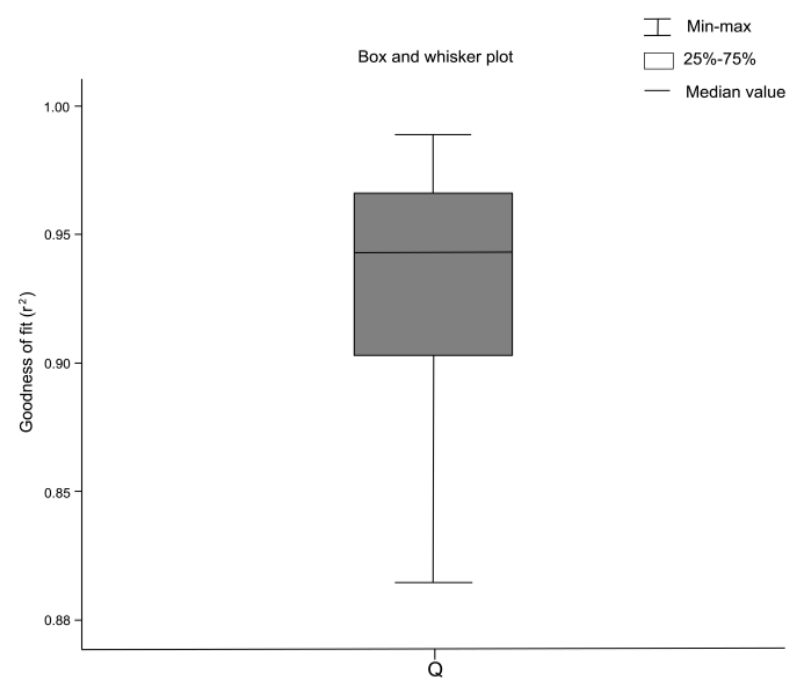

Figure 3. Box and whisker plot for the goodness of fit $\left(r^{2}\right)$ of the polynomial function for all subjects for the asphericity (Q).

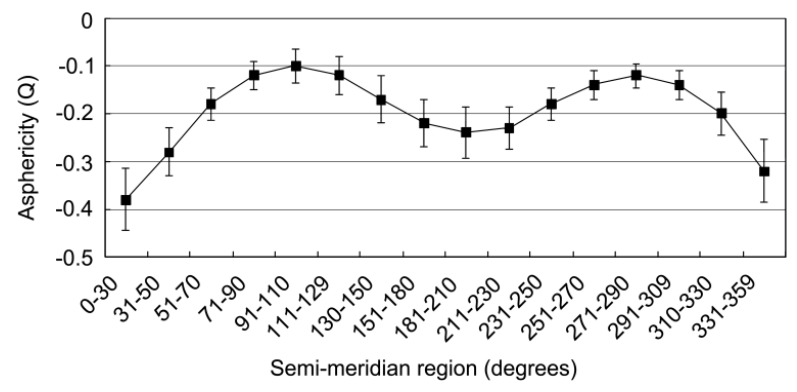

Figure 4. Variation in asphericity as a function of semimeridian region of anterior corneal surface for all subjects.

\section{Construction of a 3d Model of Corneal Shape}

\subsection{Rotation of the Coordinate System}

A new coordinate system ( $X^{\prime} O Y^{\prime}$ ) is obtained by rotating the original coordinate system ( $X O Y$ ) $\theta$ degree in the counter clockwise direction. $P$ is an arbitrary point in the coordinate system with $P(x, y)$ in the original coordinate system and $P\left(x^{\prime}, y^{\prime}\right)$ in the new coordinate system. We can obtain the following coordinate rotation formula:

$$
\left\{\begin{array}{l}
x^{\prime}=y \sin \theta+x \cos \theta \\
y^{\prime}=y \cos \theta-x \sin \theta
\end{array}\right.
$$

\subsection{The Parametric Representation of the Equations of the Corneal Meridian Section}

We set the angle between a given corneal meridian section and the $X O Z$ plane is $\theta$ degree. A new coordinate system $(\bar{X} O \bar{Y})$ is obtained by rotating the original coordinate system $(X O Y)-\left(90^{\circ}-\theta\right)$ degree in the coun- 
ter clockwise direction around the $Z$-axis. Thus, the $\bar{Y} O Z$ plane can be coincided with the corneal meridian section in the new coordinate system $(\bar{X} O \bar{Y})$. The equations of the corneal meridian section in the new coordinate system $(\bar{X} O \bar{Y})$.are as follows:

$$
\left\{\begin{array}{l}
\bar{X}=0 \\
\bar{y}^{2}=a_{1} z+a_{2} z^{2}=2 r_{0} z-(1+Q) z^{2}
\end{array}\right.
$$

where $(\bar{x}, \bar{y})$ are the coordinates of the new coordinate system $(\bar{X} O \bar{Y})$.

Then by substituting $-\left(90^{\circ}-\theta\right)$ into $\theta$ given in the formula (6), we obtain the following coordinate rotation equations of our corneal model:

$$
\left\{\begin{array}{l}
\bar{x}=x \sin \theta-y \cos \theta \\
\bar{y}=y \sin \theta+x \cos \theta
\end{array}\right.
$$

We substitute the $\bar{x}, \bar{y}$ given in Equation (7) into the Equation (8). The equations of the corneal meridian section on the original coordinate system $(X O Y)$ are as follows

$$
\left\{\begin{array}{l}
x \sin \theta-y \cos \theta=0 \\
(y \sin \theta+x \cos \theta)^{2}=2 r_{0} z-(1+Q) z^{2}
\end{array}\right.
$$

Finally, we transform the Equation (9) into the following form:

$$
\left\{\begin{array}{l}
x=\sqrt{2 r_{0} z-(1+Q) z^{2}} \cos \theta \\
y=\sqrt{2 r_{0} z-(1+Q) z^{2}} \sin \theta
\end{array}\right.
$$

\subsection{Generation of a 3D Corneal Model}

360 semi-meridians are all chosen. Every point has an $(x, y, z)$ coordinate. The $z$ is a parameter and the $z$ values of a semi-meridian are selected from $0 \mathrm{~mm}$ to 3.5 $\mathrm{mm}$ at $0.1 \mathrm{~mm}$ intervals. The $x, y$ coordinate values of every point are calculated by substituting the corresponding $Z$ value into the Equation (11), 3D corneal surface plot is generated with the Visual C++ 6.0 programming [16]. Figure 5 shows a colorized 3D surface plot of anterior corneal surface from two different perspectives for the same subject as in Figure 2. Variation of color shows semi-meridional variation of the $Q$-value with 0.02 color steps. From the top to bottom of color scale, the $Q$-value becomes more negative gradually. Figure 2 shows that the $Q$-value of each semi-meridian is negative value $(-1<Q<0)$ corresponding to the most common corneal shape (prolate ellipse) ([17]). Thus, the 3D surface plot of anterior corneal surface approximates a prolate ellipsoid shown in Figure 5.

\section{Conclusion}

In contrast to the sagittal radius of curvature $\left(r_{s}\right)$, the

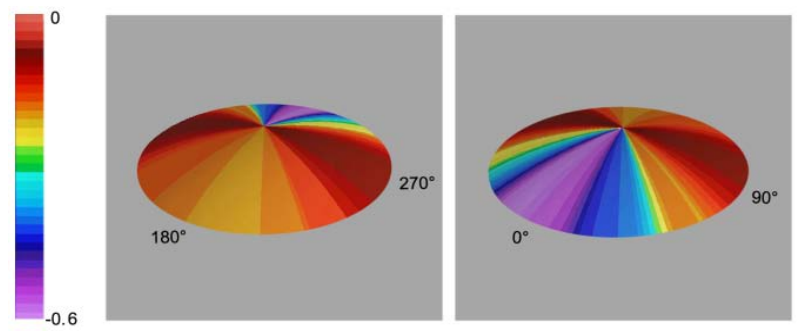

Figure 5. 3D surface plot of anterior corneal surface for the same subject as in Figure 2.

tangential radius of curvature $\left(r_{t}\right)$ is a true radius of curvature which can better represent corneal shape and local curvature changes especially in the periphery.

In this paper, we proposed a nonlinear equation of corneal asphericity $(Q)$ using the tangential radius of curvature $\left(r_{t}\right)$ on every semi-meridian. The 360 semimeridional variation of the $Q$-value was well fitted using the $7^{\text {th }}$ degree polynomial function for all subjects. We constructed a new 3D corneal model and present a more realistic model of shape of the anterior corneal surface. Our mathematical model could be helpful in the contact lens design and detection of corneal shape abnormalities, such as keratoconus or previous laser surgery.

\section{Acknowledgements}

This study was supported by grant No. 30872816 from the National Natural Scientific Foundation of China.

\section{REFERENCES}

[1] K. Scholz, A. Messner, T. Eppig, H. Bruenner and A. Langenbucher, "Topography-Based Assessment of Anterior Corneal Curvature and Asphericity as a Function of Age, Sex, and Refractive Status,” Journal of Cataract \& Refractive Surgery, Vol. 35, 2009, pp. 1046-1054. http://dx.doi.org/10.1016/j.jcrs.2009.01.019

[2] M. Guillon, D. P. M. Lydon and C. Wilson, "Corneal Topography: A Clinical Model,” Ophthalmic and Physiological Optics, Vol. 6, 1986, pp. 47-56. http://dx.doi.org/10.1111/j.1475-1313.1986.tb00699.x

[3] A. G. Bennett, "Aspherical and Continuous Curve Contact Lenses,” Optometry Today, Vol. 28 ,1988, pp. 11-14, 140-142, 238-242, 433-444.

[4] T. Y. Baker, "Ray Tracing through Non-Spherical Surfaces,” Proceedings of the Physical Society, Vol. 55, 1943, pp. 361-364. http://dx.doi.org/10.1088/0959-5309/55/5/302

[5] S. W. Cheung, P. Cho and W. A. Douthwaite, "Corneal Shape of Hong Kong-Chinese," Ophthalmic and Physiological Optics, Vol. 2, 2000, pp. 119-125. http://dx.doi.org/10.1016/S0275-5408(99)00045-9

[6] A. G. Bennett and R. B. Rabbetts, "What Radius Does the Conventional Keratometer Measure?” Ophthalmic and Physiological Optics, Vol. 11, 1991, pp. 239-247. http://dx.doi.org/10.1111/j.1475-1313.1991.tb00539.x 
[7] J. Schwiegerling and J. E. Greivenkamp, “Using Corneal Height Maps and Polynomial Decomposition to Determine Corneal Aberrations,” Optometry and Vision Science Journal, Vol. 74, 1997, pp. 906-916. http://dx.doi.org/10.1097/00006324-199711000-00024

[8] M. Dubbelman, V. A. D. P. Sicam and G. L. Van der Heijde, "The Shape of the Anterior and Posterior Surface of the Aging Human Cornea,” Vision Research, Vol. 46, 2006, pp. 993-1001. http://dx.doi.org/10.1016/j.visres.2005.09.021

[9] C. Roberts, "Characterization of the Inherent Error in a Spherically-Biased Corneal Topography System in Mapping a Radially Aspheric Surface,” Journal of Cataract \& Refractive Surgery, Vol. 10, 1994, pp. 103-111.

[10] C. Roberts, "Analysis of the Inherent Error of the TMS-1 Topographic Modeling System in Mapping a Radially Aspheric Surface,” Cornea, Vol. 14, 1995, pp. 258-265. http://dx.doi.org/10.1097/00003226-199505000-00006

[11] J. S. Chan, R. B. Mandell, D. S. Burger and R. E. Fusaro, "Accuracy of Videokeratography for Instantaneous Radius in Keratoconus," Optometry and Vision Science Journal, Vol. 72, No. 11, 1995, pp. 793-799. http://dx.doi.org/10.1097/00006324-199511000-00004

[12] L. B. Szczotka and J. Thomas, "Comparison of Axial and Instantaneous Videokeratographic Data in Keratoconus and Utility in Contact Lens Curvature Prediction," The CLAO Journal, Vol. 24, 1998, pp. 22-28.

[13] R. B. Mandell, C. S. Chiang and S. A. Klein, "Location of the Major Corneal Reference Points," Optometry and Vision Science Journal, Vol. 72, 1995, pp. 776-784. http://dx.doi.org/10.1097/00006324-199511000-00002

[14] A. Gray, "Modern Differential Geometry of Curves and Surfaces with Mathematica," 2nd Edition, CRC Press, Boca Raton, 1997.

[15] E. Kreyszig, “Differential Geometry,” Dover, New York, 1991.

[16] J. D. Foley, A. V. Dam, S. K. Feiner and J. F. Hughes, "Computer Graphics: Principles and Practice in C," 2nd Edition, Addison-Wesley, Boston, 1995.

[17] P. M. Kiely, G. Smith and L. G. Carney, "The Mean Shape of the Human Cornea,” Optica Acta, Vol. 29, 1982, pp. 1027-1040. http://dx.doi.org/10.1080/713820960 